\title{
Perceptual Factors Related to register as Organ Do- nors among Iranian Medical College Student
}

\author{
Mari Ataee ${ }^{1}$, Touraj Ahmadi-Jouybari ${ }^{1}$, Mohammad Ahmadpana ${ }^{2}$, Farzad Jalilian ${ }^{1}$, Shohreh Emdadi ${ }^{\star}$, Mohsen
}

Jalilian $^{4}$, Abbas Aghaei $^{1}$, Mohammad Mahboubi ${ }^{5}$, Marzieh Shiri ${ }^{3}$, Mahbobeh Morshedi $^{3}$

\author{
${ }^{1}$ Clinical Research Development Center, Imam Khomeini Hospital, Kermanshah University of Medical Sciences, Kermanshah, Iran \\ ${ }^{2}$ Research Institute of Behavioral Disorders and Substance Abuse, Hamadan University of Medical Sciences, Hamadan, Iran \\ ${ }^{3}$ Department of Public Health, School of Public Health, Hamadan University of Medical Sciences, Hamadan, Iran \\ ${ }^{4}$ Department of Public Health, School of Public Health, Ilam University of Medical Sciences, Ilam, Iran \\ ${ }^{5}$ Ph.D of Health Services Administration, Kermanshah University of Medical Sciences, Kermanshah, Iran
}

*correspondence should be addressed to Shohreh Emdadi, Department of Public Health, School of Health, Hamadan University of Medical Sciences, Hamadan, Iran; Tell: +988118380398; Fax: +988118380026; Email: sh.emdadi@umsha.ac.ir.

\begin{abstract}
One of the major treatments for many diseases is organ transplantation. The objective of this study was to determine factors related to register as organ donors among Iranian college student based on the theory of planned behavior. This cross-sectional study was conducted on 320 college students in Hamadan University of medical sciences. Participants filled out a standard self-administered questionnaire including the expanded theory of planned behavior components. Data were analyzed by SPSS version 21 using correlation, linear and logistic regression statistical tests at 95\% significant level. Our result showed $6.6 \%$ of participants reported registered as an organ donor card. The most of participants have reported save people life most effective factor that motivation them to register as an organ donor card. The TPB variable, accounted for $33 \%$ of the variation in the outcome measure of the intention to organ donor card registered. The best predictor for organ donor card registered was subjective norm with odds ratio estimate of 1.23 [95\% Cl: 1.04, 1.44]. Comprehensive educational programs need to emphasize on psychological factors that mediate and predict behaviors. According to the results, donor organ associated with subjective norms in community; It seems special attention to importance culture in the organ donation to save human lives utilizes by influence persons at community, could be benefit results.

Key words: Organ Donors, Students, Subjective Norm
\end{abstract}

Copyright $(\odot 2014$ Mari Ataee et al. This is an open access article distributed under the Creative Commons Attribution License.

\section{INTRODUCTION}

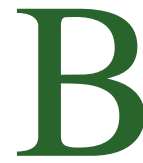

rain death is the unalterable end of brain activity (including involuntary activity necessary to sustain life) due to total necrosis of the cerebral neurons following loss of brain oxygenation (1). Brain death can be caused by any injury that stops blood and oxygen from reaching the brain. Heart attacks, strokes, brain tumor, die as a result of head injuries, blood clots and infections (2). Considering the high rate of accidents in Iran, which the ten accident one person is dead, and also in every hundred deaths one of them is brain death, we witnessed the highest numbers of brain death in Iran as compared to other countries (3). Organ donation is the process of surgically removing an organ or tissue from one person (the organ donor) and placing it into another person (the recipient). Organ transplantation is one of the great advances in modern medicine, and display a noteworthy improvement in current medical science, and it has 
promoted several patients with organ defeat (4). Currently, organ transplant, one of the most effective treatments and sometimes that is only cure for many of advanced disease; however unfortunately depending on the disease approximately $10-25 \%$ of patients who needing a transplants, without getting organ died on the waiting list (5). In addition, unfortunately, the need for organ donors is much greater than the number of people who actually donate. Every day in the United States 18 people die waiting for an organ and more than 117,000 men, women, and children await life-saving organ transplants (6). Generally amount of family consent in brain dead patients to organ donation is different in various countries; these amount during 2007 in some countries such as Hungary, Ireland, Poland, Spain, Cuba, less than 20\%, and in some of countries such as Bulgaria, Estonia, Italy, Argentina, Uruguay, Greece, between 20-50 percent, and in Panama, America, Britain, and Turkey more than 50 percent were reported (5).Understanding the factors related to register as organ donors among various social groups could be appropriate to enhance organ donation card register is effective. In this regard, two main methods have been approved to understand the factors influencing communication of the organ donation decision. One method has focused on identifying individual background factors predicting willingness to register and discuss the donation decision. The second method is the development of predictive models designed to account for variability in registration and discussion decisions (7). In this regard, several research was conducted based on theory of planned behavior to predicting factors related to register as organ donors $(7,8)$. Regarding confined of studies in our country, our TPB based study focused on exploring cognitive factors related to the register as organ donors in a sample of medical college students in Iran.

\section{MATERIALS AND METHODS}

\subsection{Participants and Procedure}

This cross-sectional study was conducted on 320 college students aged 18 to 30 years old in Hamadan University of Medical Sciences, the west of Iran, during 2012. The sample size was calculated at $95 \%$ significant level according to the results of a pilot study and a sample of 320 was estimated. Of the population of $320,302(94.3 \%)$ signed the consent form and voluntarily agreed to participate in the study, which has been approved by the research institute of behavioral disorders and substance abuse of Hamadan University of Medical Sciences, Iran. Data collection conducted after receiving approval from the relevant university ethics committee, this project was carried out and the volunteers were given the selfquestionnaire.

\subsection{Measure}

Prior to conducting the main project, a pilot study was carried out. Initially the relevant questionnaires were administered to 30 students who were similar to study population in order to estimate the duration of the study conduction and to evaluate the reliability of the questionnaire. Estimated reliability using alpha Cronbach coefficient for each TPB constructs questionnaire were as follows: attitude $(\alpha=0.73)$; subjective norms ( $\alpha$ $=0.67)$; perceived behavior control $(\alpha=0.74)$ and behavioral intention $(\alpha=0.79)$. The variables assessed in this study included: Background data collected were: age (years), level of education (BSc, MSc, MD or MDD), marital status (single or married), Job (just student or employee), Live in Dormitory (yes or no), Native of Hamadan (yes or no).

\subsection{Theoretical Framework}

The theory of planned behavior (TPB) was proposed by IcekAjzen in 1985. According to the TPB, the primary determinants of future behavior are one's intention to perform the behavior and the subjective perception of having control over behavior (perceived behavioral control - PBC). In turn, intentions are predicted by three variables: (a) Attitudes are a person's positive or negative evaluation of performing the focal behavior, (b) Subjective norms (SN) are a person's perception of other people's opinion regarding behavioral performance and (c) PBC refers to a person's sense of control over performing the behavior under study. When $\mathrm{PBC}$ is a reflection of actual control over behavioral performance, it is expected that it will predict behavior directly (9). TPB scale was designed based on standard questionnaires, and included 19 items under four constructs including (a) attitude; (b) subjective norms; (c) perceived behavioral control; (d) behavioral intention $(7,8)$. Five items were designed to measure attitude toward to register as organ donors (e.g., I think register as an organ donor could be saved other human life). Five items were designed to measure subjective norms to register as organ donors (e.g., those people who are important to me would want me to be registered as an organ donor card). Five items 
were designed to perceived behavioral control toward to register as organ donors (e.g., I am confident that I could talk to my family about my donation decision). Four items were designed to evaluate intention toward to register as organ donors (e.g., I intend to register as an organ donor card in the next months). In order to facilitate participants' responses to the items, all items were standardized to a 5-point Likert scale, ranging from 1 (strongly disagree) to 5 (strongly agree). Data were analyzed by SPSS version 21 using appropriate statistical tests including Correlation, linear and logistic regression at 95\% significant level.

\section{RESULTS AND DISCUSSION}

The mean age of respondents was 21.33 years [SD: 2.30], ranged from 18 to 30 years. More details of demographic characteristics of the participants are shown in Table 1.

\begin{tabular}{|c|c|c|}
\hline Variables & Number & Percent \\
\hline \multicolumn{3}{|c|}{ Age group (year) } \\
\hline $18-21$ & 177 & 58.6 \\
\hline $22-25$ & 108 & 35.8 \\
\hline $26-30$ & 17 & 5.6 \\
\hline \multicolumn{3}{|l|}{ Sex } \\
\hline Male & 203 & 67.2 \\
\hline Female & 99 & 32.8 \\
\hline \multicolumn{3}{|c|}{ Living in Dormitory } \\
\hline Yes & 175 & 57.9 \\
\hline No & 127 & 42.1 \\
\hline \multicolumn{3}{|l|}{ Faculty } \\
\hline Medical & 49 & 16.2 \\
\hline Dentist & 33 & 10.9 \\
\hline Pharmacology & 16 & 5.3 \\
\hline Nursing & 58 & 19.2 \\
\hline Paramedical & 47 & 15.6 \\
\hline Health & 74 & 24.5 \\
\hline Rehabilitation & 25 & 8.3 \\
\hline \multicolumn{3}{|l|}{ Marital Status } \\
\hline Single & 286 & 94.7 \\
\hline Married & 16 & 5.3 \\
\hline
\end{tabular}

Only $6.6 \%$ (20/302) of participants reported registered as an organ donor card. Participants also reported that $10.3 \%$ $(31 / 302)$ of their friends registered as an organ donor card and about $8.6 \%(26 / 302)$ of them reported that their family registered as an organ donor card. Furthermore, $21.9 \%$ (66/302) of participants have reported TV as the most effective factor that persuaded them to register as an organ donor card. The correlation between different components of theory of planned behavior is shown in Table 2. According to these results, there is a mild to moderate correlation between different components of the theory.
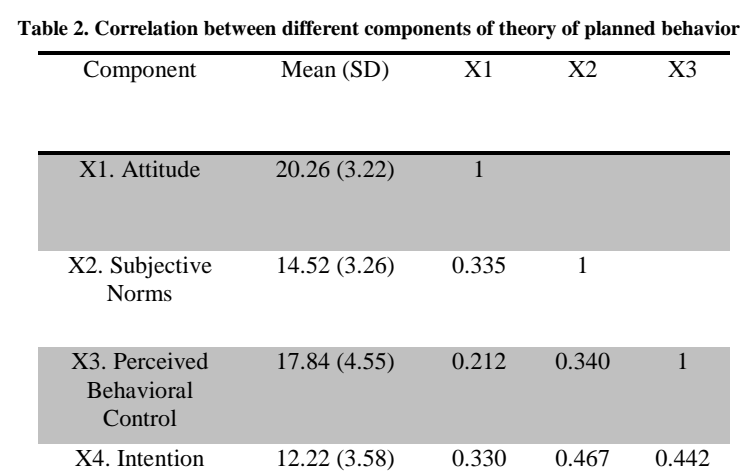

A hierarchical multiple regression analysis was performed to explain the variation in intention to organ donor card registered, using the TPB variables of perceived behavioral control, attitudes, and subjective norms. As can be seen in Table 3, were statistically significant predictors of the outcome measure. Collectively, they were accounted for $33 \%$ of the variation in intention to organ donor card registered.

\begin{tabular}{|c|c|c|c|c|c|}
\hline Variable & B & SE B & B & $\mathrm{t}$ & $\mathrm{p}$-value \\
\hline Attitude & 0.180 & 0.056 & 0.162 & 3.193 & 0.002 \\
\hline Subjective Norms & 0.341 & 0.058 & 0.310 & 5.901 & 0.000 \\
\hline Perceived behavioral & & & & & \\
\hline & 0.237 & 0.040 & 0.302 & 5.957 & 0.000 \\
\hline
\end{tabular}

According to the logistic regression analysis, subjective norms and behavioral intention were the most influential predictors on registered as an organ donor card (Table 4).

Table 4. The correlation between different components of theory of planned behavior and register as organ donors using logistic regression analysis

\begin{tabular}{lcccc}
\hline \multirow{3}{*}{$\begin{array}{l}\text { Variable } \\
\text { Odds Ratio }\end{array}$} & Lower & Upper & \\
\cline { 3 - 4 } & & & & \\
\hline Subjective Norms & 1.232 & 1.049 & 1.447 & 0.011 \\
Behavioral Intention & 1.184 & 1.006 & 1.394 & 0.042 \\
& & & & \\
\hline
\end{tabular}


Annually over than ten thousand of brain death occurs due to accidents in Iran. That is much higher than other countries; however, today, one of the most effective and sometimes the only treatment of incurable diseases is organ transplant (5). Thus, identify and analyzing factors associated with registration as organ donor among various groups is very important. Present study is discussed about factors associated with registration as organ donor among medical students using the theory of planned behavior. Our findings indicated that $6.6 \%$ of the participants were registered as organ donor. That is much lower in comparison with other countries. Hyde et al in their study which was conducted among students in Australia reported that $61 \%$ of them have donor card registration (7). Ghadi-Pasha et al in their study about organ transplantation reported that 55 percent of the doctors in Kerman city agreed with organ transplantation in brain death and 80 percent of them have a tendency to register as organ donor. Furthermore, the finding of Bormand and Asghari on study of the population over 18 years in Tehran city showed that $83.3 \%$ of those surveyed agreed with organ donation if one of their family members has suffered from brain death (10). Finding of Khadir et al study in blood donation among Iranian women showed that $24 \%$ of participants have at least one previous donation (11). Logistic regression revealed that the subjective norms and behavioral intention constructs are the strong predictors of registration as organ donor. Several studies have also shown that subjective norms are strong predictors of registration as organ donor, which is consistent with our study $(12,13)$. In this regard Stephenson suggests that subjective norms have a positive role in consent for registration as organ donor (14). Hyde et al in their study that was conducted in Australia during 2009 reported that the Subjective norms have an effective role in the decision of registration as organ donor (7). In previous studies on blood donation by Lemmens, and Myers, the effect of subjective norm on decision making in blood donors has been reported $(12,15)$. Reference groups can affect the social behavior of individuals and influence on their attitude and can act as sources for confirming a positive social behavior. It also plays an important role in the engaging in particular behaviors (16). Our findings indicated that individuals behave according to the culture and the important people in their life. Thus, culture and utilization of effective social channels can have useful effects on registration as or- gan donor in public population. According to our finding, attitude and perceived behavioral control did not a strong predictor of registration as organ donor. Stephensonin their study reported that attitude have positive role in consent people for organ donation (14). Despite of the high level of student attitude toward organ donation, According to result of present study, organ donation attitude has no significant relation with registration as organ donor. It can be demonstrated that this behavior can be influenced by other factors. Our findings reported in this study have certain limitations, such as: First, data collected by questionnaire, which is usually prone to recall bias. Second, study conducted on medical students, recommended that future studies conduct on different social groups to provide a more detailed. Third, data collection based on one of behavioral theory, we suggested application of other health behavior models or an integrated model to assess factors related to registration as organ donor.

\section{CONCLUSION}

According to the study, subjective norms and behavioral intention were strong predictors of registration as organ donor among college students. The constructs of the theory of planned behavior could predict $33 \%$ of the variance in behavioral intention in registration as organ donor.

\section{ACKNOWLEDGMENT}

This research was supported by the research institute of behavioral disorders and substance abuse of Hamadan University of Medical Sciences, Iran. We would like to thank Deputy of Research of Hamadan University of Medical Sciences for financial support of this study.

\section{AUTHORS CONTRIBUTION}

This work was carried out in collaboration between all authors.

\section{CONFLICT OF INTEREST}

Authors have declared that no conflict interests exist.

\section{REFERENCES}

1. Randell T. Medical and legal considerations of brain death. Acta anaesthesiologica scandinavica. 2004;48(2):139-44.

2. Lovasik D. Brain death and organ donation. Critical care nursing clinics of North America. 2000;12(4):531-8. 
3. Jalilian F, Shiri M, Mirzayi Alavijeh M, Morshedi M, Zinat Motlagh F. The Relationship Between Subjective Norms and Registered Volunteer Organ Donors Among Students of Hamadan University of Medical Sciences. SJFM. 2012;17(4):217-24.

4. Wang W, Tian H, Yin H, Liu H, Zhang X. Attitudes toward organ donation in China. Chin Med J. 2012;125(1):56-62.

5. Vishteh HRK, Ghorbani F, Ghobadi O, Shafaghi S, Barbati ME, Louyeh AR, et al. Causes and follow-up outcomes of brain dead patients in Shahid Beheshti University of Medical Sciences hospitals. Pajoohandeh Journal. 2010;15(4):171-8.

6. Kaur M, editor Organ donation and transplantation in Singapore. Transplantation proceedings; 1998: Elsevier.

7. Hyde MK, White KM. To be a donor or not to be? Applying an extended theory of planned behavior to predict posthumous organ donation intentions. Journal of Applied Social Psychology. 2009;39(4):880-900.

8. Hyde MK, White KM. Are organ donation communication decisions reasoned or reactive? A test of the utility of an augmented theory of planned behaviour with the prototype/willingness model. British journal of health psychology. 2010;15(2):435-52.

9. Ajzen I. The theory of planned behavior. Organizational behavior and human decision processes. 1991;50(2):179-211.

10. Bormand MA, Asghari F. Do Tehran citizens agree with organ donation from a brain-dead family member? Iranian Journal of Medical Ethics and History of Medicine. 2012;5(1).
11. Khadir M, MAGHSOUDLOU M, Gharah Baghian A, Danandeh E, Faghih H, Vafaiyan V, et al. The evaluation of the attitude of Iranian women towards blood donation. Blood (Khoon). 2004.

12. Lemmens K, Abraham C, Hoekstra T, Ruiter R, De Kort W, Brug J, et al. Why don't young people volunteer to give blood? An investigation of the correlates of donation intentions among young nondonors. Transfusion. 2005;45(6):94555.

13. Lemmens K, Abraham C, Ruiter R, Veldhuizen I, Dehing C, Bos A, et al. Modelling antecedents of blood donation motivation among non donors of varying age and education. British journal of psychology. 2009;100(1):71-90.

14. Stephenson MT, Morgan SE, Roberts-Perez SD, Harrison $\mathrm{T}$, Afifi $\mathrm{W}$, Long SD. The role of religiosity, religious norms, subjective norms, and bodily integrity in signing an organ donor card. Health Communication. 2008;23(5):436-47.

15. Amponsah-Afuwape S, Myers L, Newman S. Cognitive predictors of ethnic minorities' blood donation intention. Psychology, health \& medicine. 2002;7(3):357-61.

16. Kafashpoor A, Mortazavi S, Hasani MS. Application of social marketing concept in encouraging voluntary blood donors using theory of planned behavior. Scientific Journal of Iranian Blood Transfusion Organization. 2012;9(1). 